\title{
Clinical and Translational Imaging: the making of an international journal
}

\author{
Giovanni Lucignani
}

Published online: 14 March 2014

(C) Italian Association of Nuclear Medicine and Molecular Imaging 2014

\begin{abstract}
Briefly after resting my weary body, I resumed walking through the desert slope, so that the firm foot was always lower.
\end{abstract}

\section{Dante Alighieri, Inferno, I, 28-30}

Around four years ago, the Board of the Italian Association of Nuclear Medicine (AIMN) began to examine the possibility of launching a new journal as its official organ. The members' commitment to this new project was strengthened by their awareness of the value of this strategic choice. The idea was to develop an international journal with a global reach that would strengthen the presence of AIMN within its international scientific community of reference, that of molecular imaging. To this end, the involvement of an internationally renowned publisher, equally committed to the field of diagnostic imaging, was felt to be crucial.

The founding of the new journal in partnership with Springer thus marked a crucial change in the editorial policy of AIMN. The journal was given a clear mission to provide reviews on clinically relevant topics and research issues in the field of imaging, focusing on developments that are in the process of becoming clinically applicable. Thus, Clinical and Translational Imaging publishes invited reviews that provide in-depth and methodologically sound

\section{G. Lucignani}

Department of Biomedical Sciences and Technologies and Center of Molecular and Cellular Imaging (IMAGO),

University of Milan, Milan, Italy

\section{G. Lucignani ( $\square)$}

Department of Diagnostic Services, Unit of Nuclear Medicine, San Paolo Hospital, Via Antonio di Rudinì 8,

20142 Milan, Italy

e-mail: giovanni.lucignani@unimi.it analyses of the results of basic science and clinical research relevant to the diagnosis and care of patients. Each bimonthly issue, edited by two guest editors, is an updated and readily accessible "e-booklet" that summarizes, through key papers by selected basic and clinical scientists, the state of the art of the topic being covered. This strategy, which required the preliminary identification and "recruitment" of outstanding scientists in each of the fields and sub-fields of interest, ensures high quality and, through a combination of both innovative and well-established procedures, a valid overview of the chosen topic.

Today, at the start of the second year of publication, it is time to take stock of the situation.

First of all, it is important to point out that the success of any scientific journal depends on the valuable support of scientists willing to devote part of their time to improving the quality of scientific literature. In this regard, we feel that the results of this first year reflect our aim of ensuring high-quality content, which is an essential prerequisite for achieving a higher profile and greater recognition from the scientific community and thus for impacting on clinical practice. To guarantee these high standards of quality and help this journal become an authoritative and selective reference tool, invited papers go through a strict peerreview process. In the course of 2013 about 250 scientists were invited to participate in the peer-review process, and around 100 of them delivered valuable and detailed comments within usually tight deadlines. In particular, the contribution of the Editorial Board, composed of a panel of renowned experts with high citation records, has been outstanding. In addition to reviewing single papers, many of them have been or are actively involved as guest editors of published and forthcoming monothematic issues.

As regards the authors, it is worth noting that although the journal, in this start start-up phase, has enjoyed the 
particularly enthusiastic support of Italian scientists of international renown, contributions from non-Italian authors have also been numerous (accounting for more than $60 \%$ of all submitted papers) and the involvement of the international community is set to grow progressively.

To support authors during the submission process and to align our journal with the most established international periodicals, the peer-review process is managed via Editorial Manager. This system considerably reduces time between manuscript submission and acceptance. The average peer-review time of our journal was 75 days in 2013 and the average number of reviewers per manuscript was 3.2. Of the forty-three manuscripts published, six were accepted without revision, while twenty-two required minor revision and fifteen major revision.

A successful journal also requires a supportive structure and organization. The work of the authors, the reviewers and the board would not be possible without the efficient work of the editorial office, and the knowledge that we can rely on the drive and motivation of an international science publisher.

The six issues published during the first year contained mostly narrative review articles. The range of topics covered illustrates the journal's varied scope.

In keeping with the aim of delivering information on cutting-edge topics, the first issue of Clinical and Translational Imaging was devoted to the use of PET/MRI [1]; in this issue the authors covered the technical and methodological aspects of PET/MRI/MRS and their applications in oncology, radiotherapy treatment planning, and brain and cardiac imaging. The topic of hybrid imaging was also covered in 2013, in relation to cardiac [2] and thyroid imaging [3].

The daily activity of clinical nuclear medicine is, of course, driven not only by technological developments, but also by the possibility of using multiple radiopharmaceuticals in combination. For this reason, issue six highlighted the opportunities offered by the concurrent use of different radiopharmaceuticals [4].

Furthermore, since nuclear medicine approaches to the patient are constantly being reviewed in the light of multidisciplinary research, technological developments and patient-oriented guidelines, the third issue looked at the current approaches in thyroid imaging, which is one of the most traditional imaging fields [5].

Finally, although nuclear medicine methods are used mainly for diagnostic purposes, they are also essential research tools which are increasingly appreciated and refined as such. This is true of PET in Alzheimer's disease research, as highlighted in the fourth issue [6], and in cardiovascular research, as shown in the fifth, which looked in particular at the role of imaging in assessing the impact of diabetes, vascular inflammation and vascular dysfunction on the development of coronary artery disease.
All the issues for the next twelve months have been planned and the respective guest editors have already been invited. They will deal with translational imaging and therapy in cancer, nuclear medicine therapy of liver lesions, methodological and clinical issues in PET quantification, amyloid imaging, and the imaging of lung disease. Other guest editors are being contacted for the 2015 issues in the pipeline.

To further increase the quality of the output, the Clinical and Translational Imaging board has also begun inviting systematic reviews with or without meta-analysis. The value of this approach was already addressed in the second issue of the journal [7].

In order to maximize article visibility, all articles published in 2013 and 2014 are freely accessible to users online: we endeavor to publish articles online three weeks after acceptance and they are immediately citable through their DOI numbers. In the period between February and December 2013, the article downloads numbered approximately 8,000, a rate comparable with those of other established journals in this field. The analysis of the main bibliometric indexes, at the end of 2013, indicates an h-factor of 4 and a virtual Immediacy index of 0.3 , not including self-citations which, based on the 2012 JCR for the subject area of Radiology, Nuclear medicine and Molecular Imaging, is in line with an estimated Impact Factor close to 1.8.

Clinical and Translational Imaging papers have been disseminated on the global market through Springer's online platform which includes more than 5.6 million documents and has more than two hundred million accesses each year; all colleagues, the world over, interested in the field of diagnostic molecular imaging and therapy can now download papers published in the journal directly from SpringerLink (http://link.springer.com), allowing easy and immediate access. Moreover, on publication of each issue, a Table of Contents Alert is delivered to an increasing number of registered scientists and clinicians informing them of the availability of the papers progressively made available online.

In short, what was, just four years ago, still an idea is now an ongoing project that is delivering its first measurable results in terms of visibility and citations, most likely based on scientific content. It is our hope that the work we are doing, providing clinically sound and useful information, will eventually contribute to better quality of care for patients. This goal will be achieved as long as our reviews continue to be aimed at bridging the gaps between basic and clinical sciences and the information delivered in the published articles is based not on personal experience alone but also on methodical data digging and critical evaluation of the literature.

All those familiar with the field of science publishing know that results need to be consolidated before a 
publication receives the "blessing" of the main journal ranking institutes. We have good reason to believe that Clinical and Translational Imaging will go from strength to strength over the next few years, and win the approval of the record keeping institutes-a must in today's "publish or perish" world.

\section{G. Lucignani \\ Editor-in-Chief}

\section{References}

1. Ratib O (2013) PET/MRI: a new era in multimodality molecular imaging. Clin Transl Imaging 1(1):5-10

2. Gimelli A, Liga R (2013) Clinical applications of multimodality cardiac imaging. Clin Transl Imaging 1(5):297-304
3. Xue YL, Qiu ZL, Perotti G, Salvatori M, Luo QY (2013) ${ }^{131}$ I SPECT/CT: a one-station imaging modality in the management of differentiated thyroid cancer. Clin Transl Imaging 1(3):163-173

4. Mariani G, Strauss HW (2013) Multiagent imaging: from the pathophysiologic basis to clinical characterization and radionuclide therapy. Clin Transl Imaging 1(6):363-364

5. Luster M, Weber T, Verburg FA (2013) Changes and open issues in the management of differentiated thyroid carcinoma: an overview. Clin Transl Imaging 1(3):145-148

6. Herholz K, Boecker H, Nemeth I, Dunn G (2013) FDG PET in dementia multicenter studies and clinical trials. Clin Transl Imaging 1(4):261-270

7. Treglia G, Sadeghi R (2013) Meta-analyses and systematic reviews on PET and PET/CT in oncology: the state of the art. Clin Transl Imaging 1(2):73-75 